\title{
Magnetism and correlations in fractionally filled degenerate shells of graphene quantum dots
}

\author{
A. D. Güçlü, ${ }^{1}$ P. Potasz ${ }^{1,2}$ O. Voznyy,${ }^{1}$ M. Korkusinski, ${ }^{1}$ and P. Hawrylak ${ }^{1}$ \\ ${ }^{1}$ Institute for Microstructural Sciences, National Research Council of Canada, Ottawa, Canada \\ ${ }^{2}$ Institute of Physics, Wroclaw University of Technology, Wroclaw, Poland
}

(Dated: October 6, 2018)

\begin{abstract}
We investigate interaction effects and magnetization in triangular graphene quantum dots using a combination of tight-binding, Hartree-Fock and configuration interaction methods. We show that electronic correlations play a crucial role in determining the nature of the ground state as a function of filling fraction of the degenerate shell at the Fermi level. A half-filled charge neutral shell leads to full spin polarization but this magnetic moment can be completely destroyed by adding a single electron.
\end{abstract}

Following the progress in the fabrication of graphene 1 , 2, 3, 4, 5] based devices, lower dimensional structures such as graphene ribbons $[6,7,8,8$, , and more recently graphene quantum dots 10, 11, 12, 13, 14] are attracting increasing attention due to their non-trivial electronic and magnetic properties. In particular, it was shown that when an electron is confined to a triangular atomic thick layer of graphene with zig-zag edges, its energy spectrum collapses to a shell of degenerate states at the Fermi level (Dirac point) [15, 16, 17, 18, 19] isolated from remaining states by a gap. The degeneracy is proportional to the edge size and can be made macroscopic. This opens up the possibility to design a strongly correlated electronic system as a function of fractional filling of the shell, in analogy to the fractional quantum Hall effect 20], but without the need for a magnetic field.

In this work, we present new results demonstrating the important role of electronic correlations, beyond the Hubbard model 15, 16, 17 and mean-field density functional theory (DFT) 17, 18]. The interactions are treated by a combination of DFT, tight-binding, Hartree-Fock and configuration interaction methods (TB-HF-CI). We show that a half-filled charge neutral shell leads to full spin polarization of the island but this magnetic moment is completely destroyed by the addition of a single electron, in analogy to the effect of skyrmions on the quantum Hall ferromagnet [21, 22, 23, 24] and spin depolarization in electrostatically defined semiconductor quantum dots [25, 26, 27, 28]. The depolarization of the ground state is predicted to result in blocking of current through a graphene quantum dot due to spin blockade (SB) [26, 28].

In order to describe the interaction effects of electrons occupying the edge states (see Fig.1), we first solve the mean-field problem using a combination of tight-binding approach with a self-consistent Hartree-Fock method for the system with empty edge states, i.e. with $N_{r e f}=$ $N_{\text {site }}-N_{\text {edge }}$ electrons, where $N_{\text {site }}$ is the number of atoms, and $N_{\text {edge }}$ is the number of edge states. After extensive algebra, we arrive at the HF Hamiltonian given by

$$
\begin{gathered}
H_{M F}=\sum_{i, l, \sigma} \tau_{i l \sigma} c_{i \sigma}^{\dagger} c_{l \sigma}+\sum_{i, l, \sigma} \sum_{j, k, \sigma^{\prime}}\left(\rho_{j k}-\rho_{j k}^{b u l k}\right)(\langle i j|V| k l\rangle \\
\left.-\quad\langle i j|V| l k\rangle \delta_{\sigma, \sigma^{\prime}}\right) c_{i \sigma}^{\dagger} c_{l \sigma}+\sum_{i, \sigma} v_{i i}^{g}\left(q_{i n d}\right) c_{i \sigma}^{\dagger} c_{i \sigma}
\end{gathered}
$$

where the operator $c_{i \sigma}^{\dagger}$ creates a $p_{z}$ electron on site "i" with spin $\sigma$. The tight-binding parameters $\tau_{i l \sigma}$ are fixed to their bulk values $-2.5 \mathrm{eV}$ for nearest neighbours, and $-0.1 \mathrm{eV}$ for next nearest neighbours. Within the HartreeFock approximation, electrons interact with each other via the density matrix $\rho=\rho^{\uparrow}+\rho^{\downarrow}$ from which we subtract the graphene bulk density matrix $\rho^{\text {bulk }}$ already present in the tight-binding term $\tau_{i l \sigma}$. In addition to the on-site interaction term, all scattering and exchange terms within next nearest neighbours, and all direct interaction terms are included in the two-body Coulomb matrix elements $\langle i j|V| k l\rangle$ computed using Slater $p_{z}$ orbitals. In order to first empty the degenerate shell and later charge it with successive electrons, we transfer electrons to a metallic gate. The electrons in the graphene island interact with the gate via the term $v_{i i}^{g}$ given by

$$
v_{i i}^{g}\left(q_{\text {ind }}\right)=\sum_{j=1}^{N_{\text {site }}} \frac{-q_{\text {ind }} / N_{\text {site }}}{\kappa \sqrt{\left(x_{i}-x_{j}\right)^{2}+\left(y_{i}-y_{j}\right)^{2}+d_{\text {gate }}^{2}}}(2)
$$

where $\left(x_{i}, y_{i}\right)$ are the coordinates of the atoms. This model assumes that the induced charge $q_{\text {ind }}=-N_{\text {edge }}$ is smeared out at positions $\left(x_{i}, y_{i}\right)$ at a distance $d_{g a t e}$ from the quantum dot.

After diagonalizing the Hamiltonian,Eq.(1), we obtain $\mathrm{TB}+\mathrm{HF}$ quasi-particles denoted by the creation operator $b_{p}^{\dagger}$, with eigenvalues $\epsilon_{p}$ and eigenfunctions $\mid p>$ shown in Fig.1. We then start filling the edge states one by one, and solve the configuration interaction problem for the $N_{\text {add }}$ added electrons using

$$
\begin{aligned}
H & =\sum_{p, \sigma} \epsilon_{p} b_{p \sigma}^{\dagger} b_{p \sigma}+\frac{1}{2} \sum_{p, q, r, s, \sigma \sigma^{\prime}}\langle p q|V| r s\rangle b_{p \sigma}^{\dagger} b_{q \sigma^{\prime}}^{\dagger} b_{r \sigma^{\prime}} b_{s \sigma} \\
& +\sum_{p, q, \sigma}\left\langle p\left|v^{g}\left(N_{a d d}\right)\right| q\right\rangle b_{p \sigma}^{\dagger} b_{q \sigma}
\end{aligned}
$$



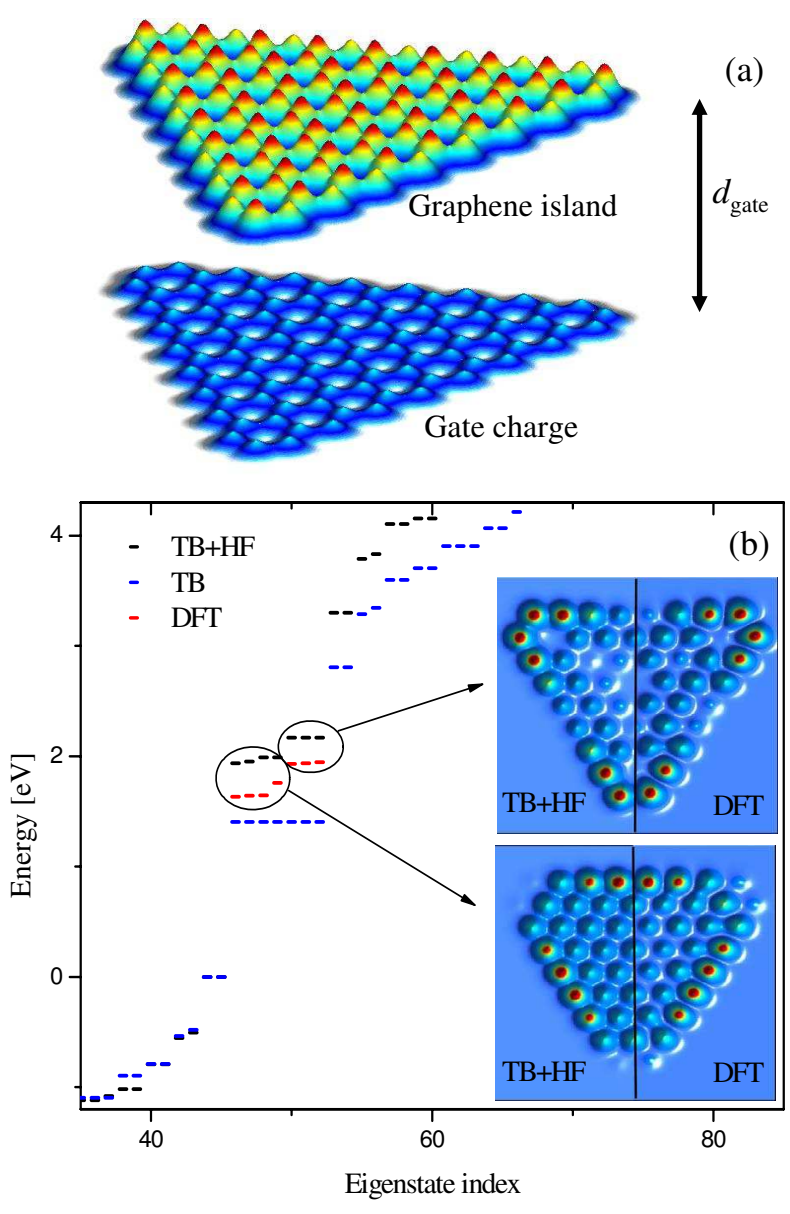

FIG. 1: (Color online) (a) Electronic density in a triangular graphene island of 97 carbon atoms where 7 electrons were moved to the metallic gate at a distance of $d_{\text {gate }}$ (b) Single particle spectrum of the structure in (a), obtained by tight-binding (TB, blue lines) and self-consistent HartreeFock (TB $+\mathrm{HF}$, black lines) methods. The 7 edge states near the Fermi level are compared to DFT results. In HartreeFock and DFT calculations 7 electrons were removed, leaving the zero-energy states empty. The dielectric constant $\kappa$ is set to 6 . Inset compares the structure of corner and side states obtained using Hartree-Fock and DFT calculations. In DFT calculations, hydrogen atoms were attached to dangling bonds.

$$
+2 \sum_{p^{\prime}}^{N_{r e f} / 2}\left\langle p^{\prime}\left|v^{g}\left(N_{a d d}\right)\right| p^{\prime}\right\rangle
$$

where the indices $(p, q, r, s)$ run over edge states, while the index $p^{\prime}$ runs over valence states (below the edge states).

Fig.1a shows the electronic density of a zig-zag edged triangular island of $N=97$ carbon atoms, separated by a distance $d_{\text {gate }}$ from a metallic gate. At zero applied voltage the island is charge neutral while applied voltage leads to removal/addition of electrons to the island. The single-particle energy spectrum obtained using nearest neighbour tight-binding method (TB, blue lines) and a combination of the next-nearest neighbour tight-binding and Hartree-Fock methods (TB+HF, black lines) is shown in Fig.1b. As was previously shown by Ezawa 16], Fernandez-Rossier and Palacios 17 and Wang, Meng and Kaxiras 18] using nearest-neighbour TB method and ab-initio DFT calculations, the linear spectrum of Dirac electrons in bulk graphene collapses to a shell of degenerate levels at the Fermi energy, well separated in energy from the valence and conduction bands. Similar to edge states in graphene ribbons [6, 7, 8, 9], zigzag edge breaks the symmetry between the two sublattices of the honeycomb lattice, behaving like a defect. Therefore, electronic states localized on the zigzag edges appear with energy in the vicinity of the Fermi level. For a $N=97$ atoms island (see Fig.1) there are $N_{\text {edge }}=7$ edge states. For the charge neutral system there is one electron per each edge state. A non-trivial question addressed here is the specific spin and orbital configuration of the electrons as a function of the size and the fractional filling of the degenerate shell of edge states. Due to the strong degeneracy, many-body effects can be expected to be important as in the fractional quantum Hall effect. Previous calculations based on the Hubbard approximation [15, 16, 17] and local spin density functional theory [17, 18] showed that the neutral system (half-filling) has its edge states polarized.

In order to study many-body effects within the charged shell of the edge states via the configuration interaction method, we first perform a Hartree-Fock calculation for the charged system of $N-N_{\text {edge }}$ electrons, with empty edge states and $N_{\text {edge }}$ electrons transferred to the gate, shown in Fig.1a. The spectrum of HF quasi-particles is shown with black lines in Fig.1b. Due to the meanfield interaction with the valence electrons and charged gate, the degeneracy of the edge states is lifted, and the electron-hole symmetry is broken. The main effect is that a group of three states is now separated from the rest by a gap of $\sim 0.2 \mathrm{eV}$. The three states correspond to HF quasiparticles localised in the three corners of the triangle. The same physics occurs in density functional calculation within local density approximation (LDA), shown with red lines in Fig.1b. Hence we see that the shell of edge states with a well defined gap separating them from the valence and conduction bands exists in the three approaches.

The wave functions corresponding to the band of nearly-degenerate edge states obtained from TB-HF calculations are used as a basis set in our configuration interaction calculations where we add $N_{a d d}$ electrons from the gate to the shell of degenerate states. In Fig.2, total spin $S$ of the ground state as a function of the filling number of the edge states is shown for different sizes of quantum dots. Two aspects of these results are particularly interesting. For the charge neutral case $\left(N_{a d d}-N_{\text {edge }}=0\right)$,for all the island sizes studied $\left(N_{\text {edge }}=3-7\right)$, the half-filled shell is maximally spin polarized as indicated by red ar- 


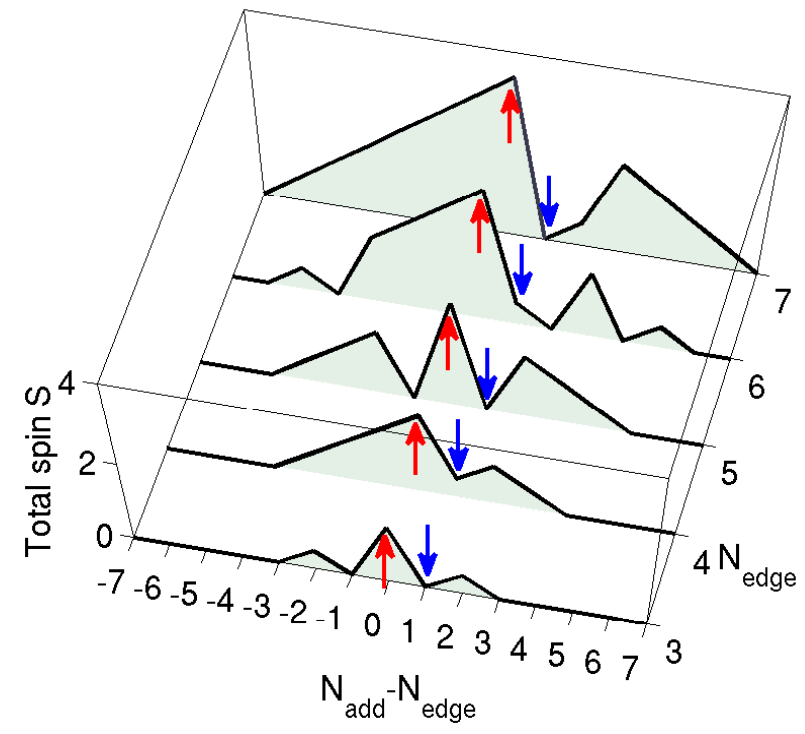

FIG. 2: (Color online) Spin phase diagram from TB-HF-CI method as a function of the size of the triangular dot characterized by the number of edge states $N_{\text {edge }}$, and the filling of the zero-energy states Nadd-Nedge. Charge neutral case corresponds to $N_{a d d}-N_{e d g e}=0$, for which the total spin of the zero-energy electrons are always maximized $\left(S=N_{\text {edge }} / 2\right.$, indicated by red arrows). On the other hand, if the quantum dot is charged by 1 electron $\left(N_{a d d}-N_{\text {edge }}=1\right)$ then the total spin has minimum value, i.e. $S=0$ if $N_{a d d}$ is even, $S=1 / 2$ if $N_{a d d}$ is odd (indicated by blue arrows).

rows. This is in agreement with our DFT calculations and reproduces previous work [17, 18]. However, the spin polarisation is extremely fragile. If we add one extra electron $\left(N_{\text {add }}-N_{\text {edge }}=1\right)$, magnetisation of the island collapses to the minimum possible value, as indicated by blue arrows in Fig.2. We note that full or partial depolarization occurs for other filling numbers but we focus here on the spin depolarisation at half-filling. The spin depolarisation was found to be insensitive to the screening of electron-electron interactions, as values of dielectric screening constant $\kappa$ between 2 and 8 led to the same behaviour of the charge neutral and single electron charged cases (not shown).

In order to illuminate the depolarization process as an electron is added to the charge neutral maximally spin polarized system, in Fig.3a,b we show the orbital occupancy of up-spin edge states at $N_{a d d}-N_{\text {edge }}=1$, for the fully polarized state $S=6 / 2$ (upper panel) and for the ground state, $S=0$, (lower panel) for the $N_{\text {site }}=97$ atoms quantum dot with 7 edge states shown in Fig.1. For the large spin $S=6 / 2$ case, the added spin up electron simply occupies the orbital 1 and its spin is opposite to the spins of other 7 electrons. However, the true ground state has $S=0$, with spin occupancy shown in the lower panel. The added electron causes electrons already present to partially flip their spin, with spin up

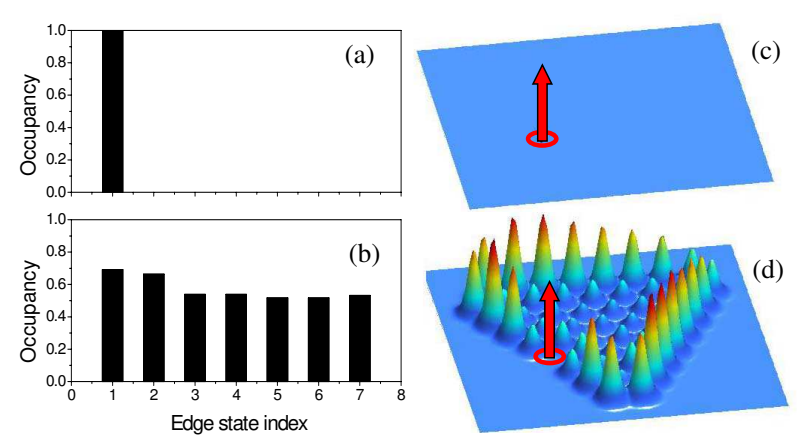

FIG. 3: (Color online) Left panel: Orbital occupancy of the 7 edge states by spin up electrons, for the charged $\left(N_{a d d}-\right.$ $N_{\text {edge }}=1$ ) system, for (a) $S=6 / 2$ and (b) $S=0$ total spin states. The ground state is $S=0$ (see Fig.2). Right panel: Corresponding spin up-up pair-correlation functions $\left\langle\rho_{\uparrow}\left(\mathbf{r}_{0}\right) \rho_{\downarrow}(\mathbf{r})\right\rangle$. The fixed spin-up electron is represented by a red arrow, and its position r0 by a red circle.

density being delocalized over all the 7 orbitals in analogy to skyrmion-like excitations in quantum dots and quantum Hall ferromagnets 21, 22, 23, 24]. The correlated nature of the $S=0$ spin depolarised ground state is illustrated by the up-up spin pair correlation functions given by $\left\langle\rho_{\uparrow}\left(\mathbf{r}_{0}\right) \rho_{\downarrow}(\mathbf{r})\right\rangle$, shown in Fig.3c,d. The location of the fixed up-spin electron at site $\mathbf{r}_{0}$ is schematically shown with an up arrow. The up-up spin correlation function for $S=6 / 2$ spin polarized system is strictly zero as there are no other spin up electrons. The spin correlation function for the spin depolarized ground state with $S=0$ shows exchange hole at $\mathbf{r}_{0}$, which extends to the nearest neighbours, and, more interestingly, for larger $\left|\mathbf{r}_{0}-\mathbf{r}\right|$, spin pair correlation function reveal a spin texture: Beyond the exchange hole there is the formation of an electronic cloud with positive magnetization which decreases and changes sign at even larger distance, again consistent with the skyrmion picture 21, 22].

Experimentally, spin properties of quantum dots can be probed using Coulomb and spin blockade spectroscopy [28]. By connecting graphene quantum dot to leads and measuring the conductance as a function of gate voltage, one obtains a series of Coulomb blockade peaks. The relative position of these peaks and their height reveal information about the electronic properties of the system as the number of electrons is increased. The amplitude of the Coulomb blockade peak is given by the conductivity $G_{i}$ of the graphene quantum dot connected to leads via atom " $i$ " [29] as shown schematically in Fig.4a. Spin and correlation effects are reflected in the weight of Coulomb blockade peak proportional to the matrix element $\left|\left\langle N+1, J^{\prime}, S^{\prime}\left|c_{i \sigma}^{\dagger}\right| N, J, S\right\rangle\right|^{2}$ which gives the transition probability from state $(N, J, S)$ to state $\left(N+1, J^{\prime}, S^{\prime}\right)$ when additional electron is added to the site " $i$ " of the graphene quantum dot from the lead. The ground state configuration $(N, J, S)$ is controlled by the 


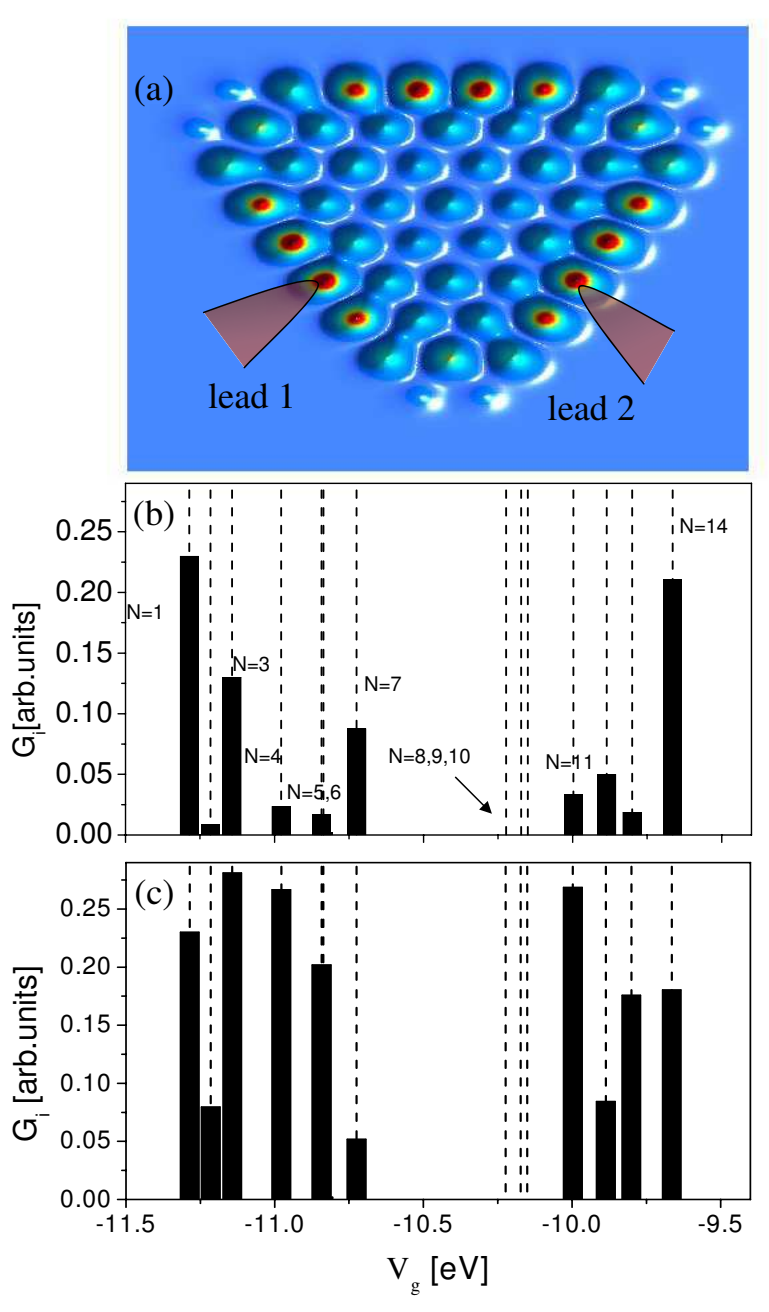

FIG. 4: (Color online) (a) Schematic representation of the graphene island connected to the leads through a side site. (b) Conductivity as a function of applied gate voltage $V_{g}$ due to an electron entering the dot through the side atom. (c) Same as (b) but without the site dependence of the incoming electron. The oscillations of the spectral weight in (c) are purely to due correlation effects and spin blockade.

gate voltage. For our model graphene quantum dot with $N=7$ degenerate zero energy states, we can add a total of 14 electrons. Hence, one expects to obtain 14 peaks. In Fig. $4 \mathrm{~b}$ some of the peaks have zero height due to spin blockade phenomenon. For instance, the transitions from $(N=7, S=7 / 2)$ states to $(N=8, S=0)$ states are spin blocked since it is not possible to change the spin of the system by $\Delta S=-7 / 2$ by adding one electron with $S=1 / 2$. Similarly, transitions from $(N=9, S=1 / 2)$ states to $(N=10, S=4 / 2)$ states are spin blocked. Besides the spin blockade, one sees a strongly oscillating feature of the spectral function heights. This is due to (a) strongly correlated nature of the states $|N, S\rangle$, and (b) specific choice of the site " $i$ ", where the lead is attached to. Here, we chose a site close to the middle of one of the sides of the triangle. The overlap of the site wavefunction is strongly dependent on the nature of the edge-states. In particular, the existence of corner states, as discussed in Fig.1, strongly affects the transition probabilities. To isolate the effect of correlation to the lead's position, in Fig.4c we plot the conductivity assuming that the weight of the site " $i$ " is a constant independent of the edge-state. As a result, the weights of spectral peaks are different, except for $N=8,9,10$ where the spin blockade occurs. These results show how to detect the spin depolarization in transport experiments. Ultimately, we show here that one can design a strongly correlated electron system in carbon based material whose magnetic properties can be controlled by applied gate voltage.

Acknowledgement. The authors thank NRC-CNRS CRP, Canadian Institute for Advanced Research, Institute for Microstructural Sciences, and QuantumWorks for support.

[1] K. S. Novoselov, A. K. Geim, S. V. Morozov, D. Jiang, Y. Zhang, S. V. Dubonos, I. V. Grigorieva, and A. A. Firsov, Science 306, 666 (2004).

[2] K. S. Novoselov, A. K. Geim, S. V. Morozov, D. Jiang, M. I. Katsnelson, I. V. Grigorieva, S. V. Dubonos, and A. A. Firsov, Nature 438, 197 (2005).

[3] Y. B. Zhang, Y. W. Tan, H. L. Stormer, and P. Kim, Nature 438, 201 (2005).

[4] S. Y. Zhou, G. H. Gweon, J. Graf, A. V. Fedorov, C. D. Spataru, R. D. Diehl, Y. Kopelevich, D. H. Lee, S. G. Louie, and A. Lanzara, Nature Phys. 2, 595 (2006).

[5] A. H. C. Neto, F. Guinea, N. M. R. Peres, K. S. Novoselov, and A. K. Geim, Rev. of Mod. Phys. 81, 109 (2009).

[6] K. Nakada, M. Fujita, G. Dresselhaus, and M. S. Dresselhaus, Phys. Rev. B 54, 17954 (1996).

[7] K. Wakabayashi, M. Fujita, H. Ajiki, and M. Sigrist, Phys. Rev. B 59, 8271 (1999).

[8] B. Wunsch, T. Stauber, F. Sols, and F. Guinea, Phys. Rev. Lett. 101 , 036803 (2008).

[9] L. Yang, M. L. Cohen, and S. G. Louie, Phys. Rev. Lett. 101,186401 (2008).

[10] B. Wunsch, T. Stauber, and F. Guinea, Phys. Rev. B 77 , 035316 (2008).

[11] J. Wurm, A. Rycerz, I. Adagideli, M. Wimmer, K. Richter, and H. U. Baranger, Phys. Rev. Lett. 102 , 056806 (2009).

[12] A. R. Akhmerov and C. W. J. Beenakker, Phys. Rev. B $\mathbf{7 7}, 085423$ (2008).

[13] F. Libisch, C. Stampfer, and J. Burgdorfer, Phys. Rev. B 79, 115423 (2009).

[14] Z. Z. Zhang, K. Chang, and F. M. Peeters, Phys. Rev. B 77, 235411 (2008).

[15] M. Ezawa, Phys. Rev. B 76 , 245415 (2007).

[16] M. Ezawa, Phys. Rev. B 77 , 155411 (2008).

[17] J. Fernandez-Rossier and J. J. Palacios, Phys. Rev. Lett. 99, 177204 (2007).

[18] W. L. Wang, S. Meng, and E. Kaxiras, Nano Letters 8, 241 (2008).

[19] J. Akola, H. P. Heiskanen, and M. Manninen, Phys. Rev. 
B 77, 193410 (2008).

[20] D. C. Tsui, H. L. Stormer, and A. C. Gossard, Phys. Rev. Lett. 48, 1559 (1982).

[21] S. L. Sondhi, A. Karlhede, S. A. Kivelson, and E. H. Rezayi, Phys. Rev. B 47, 16419 (1993).

[22] A. H. MacDonald, H. A. Fertig, and L. Brey, Phys. Rev. Lett. 76, 2153 (1996).

[23] K. Nomura and A. H. MacDonald, Phys. Rev. Lett. 96 , 256602 (2006).

[24] P. Plochocka, J. M. Schneider, D. K. Maude, M. Potemski, M. Rappaport, V. Umansky, I. Bar-Joseph, J. G. Groshaus, Y. Gallais, and A. Pinczuk, Phys. Rev. Lett. 102,126806 (2009).
[25] P. Hawrylak, Phys. Rev. Lett. 71, 3347 (1993).

[26] J. J. Palacios, L. Martín-Moreno, G. Chiappe, E. Louis, and C. Tejedor, Phys. Rev. B 50, 5760 (1994).

[27] M. Korkusinski, P. Hawrylak, M. Ciorga, M. PioroLadriere, and A. S. Sachrajda, Phys. Rev. Lett. 93 , 206806 (2004).

[28] M. Ciorga, A. S. Sachrajda, P. Hawrylak, C. Gould, P. Zawadzki, S. Jullian, Y. Feng, and Z. Wasilewski, Phys. Rev. B 61, 16315 (2000).

[29] A. D. Güçlü, Q. F. Sun, H. Guo, and R. Harris, Phys. Rev. B 66,195327 (2002). 